\title{
Multichannel analysis of correlation length of SEVIRI images around ground-based cloud observatories to determine their representativeness
}

\author{
J. Slobodda ${ }^{1}$, A. Hünerbein ${ }^{2}$, R. Lindstrot ${ }^{1}$, R. Preusker ${ }^{1}$, K. Ebell ${ }^{3}$, and J. Fischer ${ }^{1}$ \\ ${ }^{1}$ Institute for Space Sciences, Freie Universität Berlin, Carl-Heinrich-Becker-Weg 6-10, 12651 Berlin, Germany \\ ${ }^{2}$ Leibniz Institute for Tropospheric Research, Permoserstraße 15, 04318 Leipzig, Germany \\ ${ }^{3}$ Institute for Geophysics and Meteorology, University of Cologne, Pohligstr. 3, 50969 Cologne, Germany
}

Correspondence to: J. Slobodda (jennifer.slobodda@wew.fu-berlin.de)

Received: 8 April 2014 - Published in Atmos. Meas. Tech. Discuss.: 6 June 2014

Revised: 3 January 2015 - Accepted: 7 January 2015 - Published: 4 February 2015

\begin{abstract}
Images of measured radiance in different channels of the geostationary Meteosat-9 SEVIRI instrument are analysed with respect to the representativeness of the observations of eight cloud observatories in Europe (e.g. measurements from cloud radars or microwave radiometers). Cloudy situations are selected to get a time series for every pixel in a $300 \mathrm{~km} \times 300 \mathrm{~km}$ area centred around each ground station. Then a cross correlation of each time series to the pixel nearest to the corresponding ground site is calculated. In the end a correlation length is calculated to define the representativeness.

It is found that measurements in the visible and near infrared channels, which respond to cloud physical properties, are correlated in an area with a 1 to $4 \mathrm{~km}$ radius, while the thermal channels, that correspond to cloud top temperature, are correlated to a distance of about $20 \mathrm{~km}$. This also points to a higher variability of the cloud microphysical properties inside a cloud than of the cloud top temperature. The correlation length even increases for the channels at 6.2, 7.3 and $9.7 \mu \mathrm{m}$. They respond to radiation from the upper atmospheric layers emitted by atmospheric gases and higher level clouds, which are more homogeneous than low-level clouds.

Additionally, correlations at different distances, corresponding to the grid box sizes of forecast models, were compared. The results suggest the possibility of comparisons between instantaneous cloud observations from ground sites and regional forecast models and ground-based measurements. For larger distances typical for global models the correlations decrease, especially for short-wave mea-
\end{abstract}

surements and corresponding cloud products. By comparing daily means, the correlation length of each station is increased to about 3 to 10 times the value of instantaneous measurements and also the comparability to models grows.

\section{Introduction}

In our climate system clouds are one of the most important elements. They are part of the water cycle and transport large amounts of water from the oceans to the continents. Furthermore they reflect solar irradiance and thus cool the earth but also have a warming effect by blocking the outgoing longwave radiation. In this way they strongly influence the earth's energy budget (Kiehl and Trenberth, 1997). Still the full degree of cloud-radiation interactions is not fully understood so that e.g. the sign and magnitude of the impact of the specific cloud types on the net fluxes are still not known (IPCC, 2013). Especially our information on cloud formation and development as well as on time, location and amount of precipitation is incomplete. Thus weather and climate models produce clouds and precipitation slightly at the wrong time and place or overestimate their amount (e.g. Boehme et al., 2011; Crewell et al., 2008; Feldmann et al., 2008; Bouniol et al., 2010).

Additionally, the radiative transfer equation is often simplified in radiative transfer models to reduce computing time in weather and climate models by assumptions like infinite plane-parallel cloud layers. Since real clouds have a complex 
three-dimensional structure, leading to shading and brightening effects on the cloud top, and their horizontal extension is neither infinite nor homogeneous the results of radiative transfer models have some uncertainties (e.g. Welch and Wielicki, 1984; Zinner and Mayer, 2006).

Ground stations with a variety of different instruments like microwave radiometers, cloud radars and ceilometers are a good source for accurate measurements of cloud properties like vertical extent, water content, optical depth and effective radius. Those sites are used for validation studies of satellite data or forecasting models (e.g. Pfeifer et al., 2010; Greuell and Roebeling, 2009; Grützun et al., 2013). However, such stations are rare. The Cloudnet algorithm (Illingworth et al., 2007) e.g. which determines different synergy products like liquid water path (LWP), a cloud classification and rain rate from the above mentioned instruments, is only regularly calculated for Cabauw in the Netherlands, Chilbolton in southern England, Jülich, Leipzig and Lindenberg in Germany, Mace Head in Ireland, Palaiseau in France and Potenza in southern Italy. Furthermore, many instruments only examine the atmospheric column directly above, making an area-wide examination of clouds impossible. A ceilometer e.g. has a field of view of only $1.8 \mathrm{mrad}$ (Wiegner and Geiß, 2012), although the beam width of a cloud radar is wider with about $0.5^{\circ}$ (METEK, 2014) and the field of view is even larger due to its ability to scan the surroundings. This complicates comparisons both to satellite images as well as to model grid boxes with a horizontal extent. Thus an estimation of the representativeness of these spatially separated cloud observatories for their surrounding areas is desirable. Such an estimation of representativeness is also valuable for synergetic products from ground-based measurements and satellite data. The benefit of this combination was shown in Ebell et al. (e.g. 2013). These products might not only be calculated for the pixel corresponding to the ground-station, but also for the surrounding area in which the ground-based data are still representative.

There have been different approaches of estimating the correlation between different stations or the representativeness of a single station to its surroundings. Long and Ackermann (1995) e.g. examined the correlation between measurements by pyranometers at 11 locations in Wisconsin during the FIRE Project (Whitlock et al., 1990). They found that for a given measurement at one station an estimation of the measurement at a second station can be given and that the accuracy of this estimation increases for temporally averaged data in comparison to instantaneous measurements and depends on the length of the averaging interval.

Deneke et al. (2009) performed a study for two stations located in Europe. They included satellite data from the spinning enhanced visible and infrared imager (SEVIRI) on board of Meteosat second generation (MSG) into their examinations and compared cloud transmission at the ground to cloud reflection at the satellite. They showed the importance of correcting the satellite data for parallax shift caused by

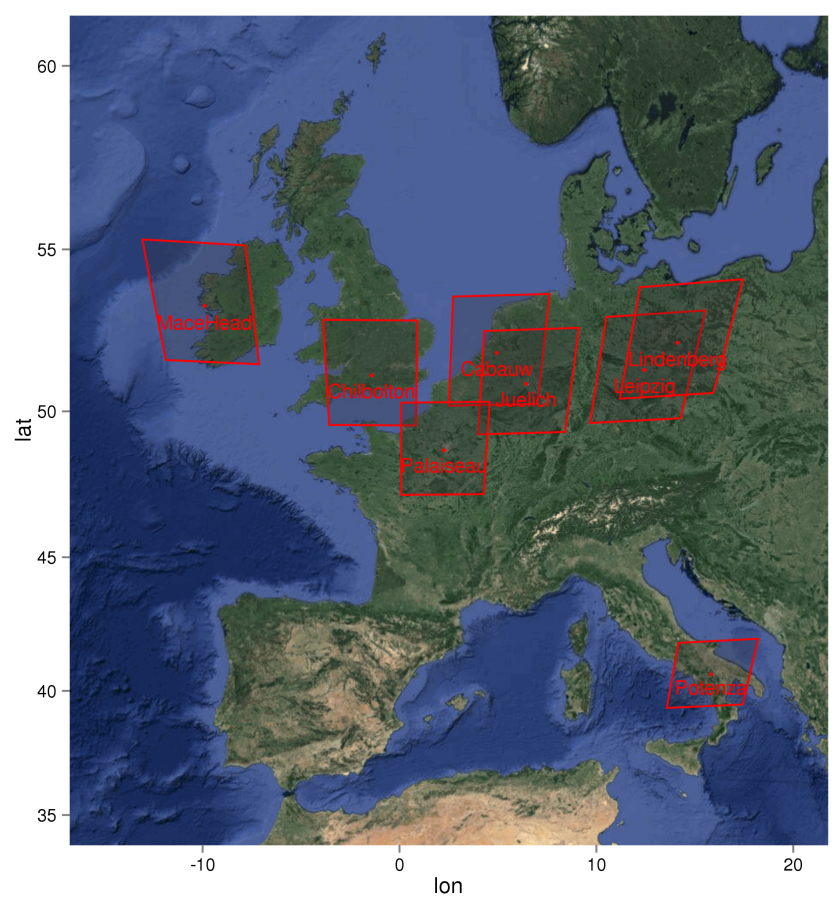

Figure 1. Map of the ground-based measurement sites and their surrounding area.

differing cloud top heights and the viewing geometry of the satellite. Due to the different spatial resolutions of groundand satellite-based data, time-averaging was needed for an optimal comparison. A 40 to $80 \mathrm{~min}$ averaging time provides best results. The correlation between satellite data around the ground station and the measurements at the station itself decreases sub-linearly.

Further error sources that arise during the validation of satellite data with ground-based measurements are discussed in Schutgens and Roebling (2009). Therefore the authors compared LWP retrievals from SEVIRI and microwave radiometers in Northern Europe. They found that the largest errors are caused by different fields of view (between visible and near infrared channels of the SEVIRI instrument as well as between satellite pixels and the ground-based measurement instruments), collocation errors due to the parallax shift and retrieval errors induced by the assumption of plane parallel clouds. The studies discussed above are based on data from time periods that are too short for climatologies. For the present study SEVIRI data from the complete year 2012 was utilized to get at least cloud types typical for all seasons though it is still not long enough for a real climatology. But this time period is sufficiently long to contain all common cloud types over Europe like pre- and post-frontal clouds, convective cumulus and boundary layer stratus. In this study only SEVIRI observations are used to understand the representativeness of the Cloudnet stations for their surroundings. Therefore the SEVIRI pixel nearest to the ground station is compared to all $101 \times 61$ pixels surrounding it (see Fig. 1). 
Thus uncertainties due to different resolutions and dimensions are minimized. We still have some differences induced by different pixel sizes around the stations. The southernmost station, Potenza, is surrounded by pixel of $\sim 15 \mathrm{~km}^{2}$ and the northernmost station, Mace Head, by pixel of $\sim 22 \mathrm{~km}^{2}$. The nearest point is defined by the clear sky geographic coordinates. These are the coordinates of the location on the earth surface the satellite sees, when no clouds are present. Because of the viewing geometry, a (high) cloud located above the same place is related to another location (parallax shift). The parallax shift is neglected because for a homogeneous cloud cover the shift is similar for all pixels and the reference point is not a fixed point on the earth. Even for inhomogeneous scenes with large cloud top heights of $\sim 10 \mathrm{~km}$ next to cloud free pixels, the parallax shift is approximately $5 \mathrm{~km}$ which corresponds to one pixel. For an area of 600 pixels this is neglectable.

In Sect. 2 the methodology is described in more detail including an overview of the data used. The results are discussed in Sect. 3. In Sect. 4 an examination of the influence of timescales follows. Conclusions and outlook are summarized in Sect. 5.

\section{Data and methodology}

This study is based on data obtained from the SEVIRI instrument on board Meteosat-9, a geostationary satellite operated by EUMETSAT. Meteosat-9 was launched in December 2005 and became operational in April 2007. It replaced Meteosat- 8 at position $0^{\circ} \mathrm{W}$. The images are taken by scanning the earth from east to west (each line) by using the spin of the satellite around its own axis and from south to north by stepwise rotating the scan mirror. The repetition cycle for a whole scan is $15 \mathrm{~min}$.

In the time period examined during this study, Meteosat-9 provided images of the full earth disk around Africa and Europe every $15 \mathrm{~min}$. Over Europe the pixel size of SEVIRI is about $3 \times 5 \mathrm{~km}$ except for the high-resolution visible channel (HRV) which was not used for our examinations. The other twelve channels range from 0.6 to $13.4 \mu \mathrm{m}$. The first three channels $(0.6,0.8$ and $1.6 \mu \mathrm{m})$ were considered in units of reflectance, the others in units of brightness temperature (BT) (more information about the SEVIRI channels can be obtained from Schmetz et al., 2002).

For each of the eight stations where the Cloudnet classification (Illingworth et al., 2007) is calculated regularly, a corresponding cutout of $101 \times 61$ pixels which corresponds to approximately $300 \mathrm{~km} \times 300 \mathrm{~km}$ (displayed in Fig. 1) is examined. This area is smaller than the correlation radii of $400-500 \mathrm{~km}$ found by Cahalan et al. (1982) for infrared radiances measured by the NOAA satellite over the mid-latitude Pacific Ocean. They define the radius according to where the correlation drops to $1 / e \approx 0.4$. This value seems to be too small to describe representativeness and instead a threshold of 0.9 was defined for the present study. This causes a reduction of the correlation length so that the chosen cutouts are sufficiently large. Additionally radiances in the visible spectral region were included which have a smaller correlation radius than infrared radiation (which will be shown in Sect. 3). Furthermore this study includes not only the radius at a certain correlation but also the correlation at certain distances that correspond to grid sizes of different forecast models.

The examinations are limited to day-time images with a solar zenith angle lower than $80^{\circ}$. The infrared channels might also be examined for night time, but for channels that measure solar reflections, night-time observations are useless. For the sake of equality night-time images are left out for all channels.

Two different types of cloudiness have been examined during this study divided by the cloud cover over the respective examination area. Using the cloud mask developed at the Institute for Space Sciences of the Freie Universität Berlin (FUB-cm, Reuter et al., 2009), scenes with nearly full cloud cover and approximately half covered scenes were selected. The cloud mask algorithm is based on a neural network and is supposed to be quite conservative. That means a pixel declared as "cloudy" is most likely really cloudy, but especially thin clouds are quite often missed (Hamann et al., 2013). The fully covered scenes contain mostly large-scale clouds especially those at frontal zones and boundaries between air masses, but also low stratus fields or high fog, and large convective cells e.g. at squall lines. Partly cloudy scenes consist either of small-scale cumulus, like fair weather cumulus, or post frontal convection or the scene is covered half by a frontal zone and the other half is more or less cloud free. Since the interest of this study lies in the representativeness of ground-based measurements of clouds and cloud properties, cloud-free pixels are masked out in both cases to diminish the influence of the earth's surface.

Cloudy time steps are defined by a horizontal mean over the $101 \times 61$ pixel area with a cloud cover larger than $90 \%$. Half covered scenes are defined by a cloud cover between 40 and $60 \%$. The cloud-free pixels in both cases are ignored for the following analysis by setting them to $\mathrm{NaN}$ and calculating the correlations only for pairwise complete observations. The number of fully and half cloudy cases in comparison to all other cases can be obtained from Fig. 2. Apart from the different numbers of cases for each station, also the type of clouds observed over each region differs. Potenza in the south of Italy e.g. has the least cloud cover of all stations. If there are clouds, these are often cumulus clouds caused by orography while the surrounding Mediterranean Sea is cloud free. Totally covered scenes are mostly related to low pressure systems passing directly over Italy. Mace Head at the west coast of Ireland is seldom completely cloud free. It lies often directly beneath cyclones moving from the Atlantic Ocean towards continental Europe. The frontal zones corresponding to these cyclones often pass over the other stations. Mostly they influence all stations, but there are also 


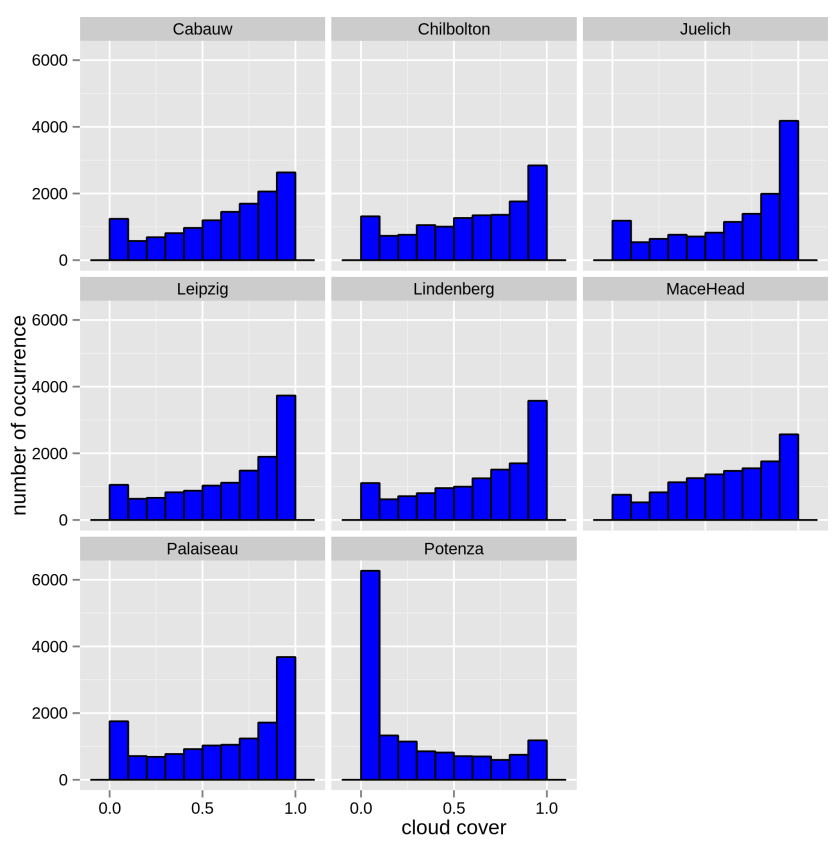

Figure 2. Histogram of mean cloud cover from day-time SEVIRI images of the area surrounding each ground station.

cases where the front dissolves before it reaches Leipzig and Lindenberg in the eastern part of Germany. There are less fully cloudy scenes in the vicinity of ocean waters causing large differences between stations near the coast and those more upcountry. The North Sea e.g. is more often cloud free than the adjacent land. Thus totally covered scenes are less frequent for Cabauw and Chilbolton than for Jülich and Palaiseau. Additionally more large convective systems passed over Palaiseau in the observed year than over the other stations. Even between Leipzig and Lindenberg, which are close to each other and both are characterized by continental weather, some differences occur. Lindenberg is located closer to the Baltic Sea and especially in the winter months stratiform clouds are often transported from there to the south and lead to a totally covered area around Lindenberg, but not over Leipzig.

For each pixel the correlation of time series of either fully or half covered scenes, determined by using a cloud mask, to the corresponding series of the nearest pixel to the respective ground station is calculated. The distance to the reference pixel is calculated from the latitude and longitude values of the pixel positions. The relationship between distance (dist) and correlation $\rho$ allows for a description by the following: $\rho=1-\frac{\mathrm{dist}^{b}}{a}$. This formula meets the requirement $\rho($ dist $=0 \mathrm{~km})=1$ and fits to convex as well as to concave shapes of the relation depending on whether coefficient $b$ is smaller or larger than 1 . The coefficient $a$ describes the intensity of the decrease of correlation with distance. While $b$ has no unit, $a$ has the unit $\mathrm{km}^{b}$, which is difficult to interpret. dist $0=a^{1 / b}$ is the theoretical distance where the corre- lation becomes zero and has the unit $\mathrm{km}$. Using this correlation length enables us to directly compare different spectral channels and geographic locations. However, dist $t_{0}$ is in all cases much larger than the range observed in this study and the information content for distances larger than approximately $200 \mathrm{~km}$ is questionable due to the high variability of clouds and their properties. Instead values for dist $_{0.9}$ with $1-$ dist $_{0.9}^{b} / a=0.9$ were calculated. The magnitude of this quantity seems suitable for a comparison of all channels and stations and a correlation of 0.9 acceptable for the definition of representativeness. Additionally the correlation at distances of the size of different model grid-boxes is compared. These sizes are $2.8 \mathrm{~km}$ for COSMO-DE, $7 \mathrm{~km}$ for COSMOEU as well as 28 and $70 \mathrm{~km}$ respectively for the Global Forecast System (GFS; grid-size is enlarged for forecast times longer than one week). This allows an estimation of the uncertainties that arise for comparisons between model data and ground-based measurements because of different horizontal resolutions.

\section{Results}

As an example Figs. 3 and 4 show the correlation around Jülich for each channel for totally cloud covered and half covered scenes, respectively. As expected, the correlation decreases with increasing distance in each channel. The decrease is strongest for the visible channels. This indicates a high variability in cloud physical parameters such as optical depth and effective radius since these channels are strongly depending on them, though it may be overlayed by effects of the three-dimensional structure of the cloud tops. In this case the cloud parameters might be more homogeneous than the results suggest. As expected, the decrease is even stronger for inhomogeneous scenes due to possible combinations of different cloud types with varying properties in the scene. The highest correlations are achieved for channels 6.2 and $9.7 \mu \mathrm{m}$. Both channels are strongly influenced by water vapour and ozone absorption, respectively. This indicates that both atmospheric components are horizontally more homogeneous than clouds. $7.3 \mu \mathrm{m}$ is also responding to water vapour but in lower parts of the atmosphere as can be seen in the weighting functions of the SEVIRI channels in Schmetz et al. (2002). Therefore it is more strongly influenced by medium to high clouds, resulting in lower correlation than for the $6.2 \mu \mathrm{m}$ channel. For these reasons the difference between totally clouded scenes and half covered ones is less than for other channels. The other infrared channels correspond mostly to cloud top temperature. The fully covered scenes are most likely covered with one large cloud layer with a more or less homogeneous top height. Due to the relatively high homogeneity the correlations decrease slowly. Still larger inhomogeneities might arise from multilayer clouds with edges in one layer. For partly covered scenes there are two possibilities. Either one large cloud layer with an edge covers half 

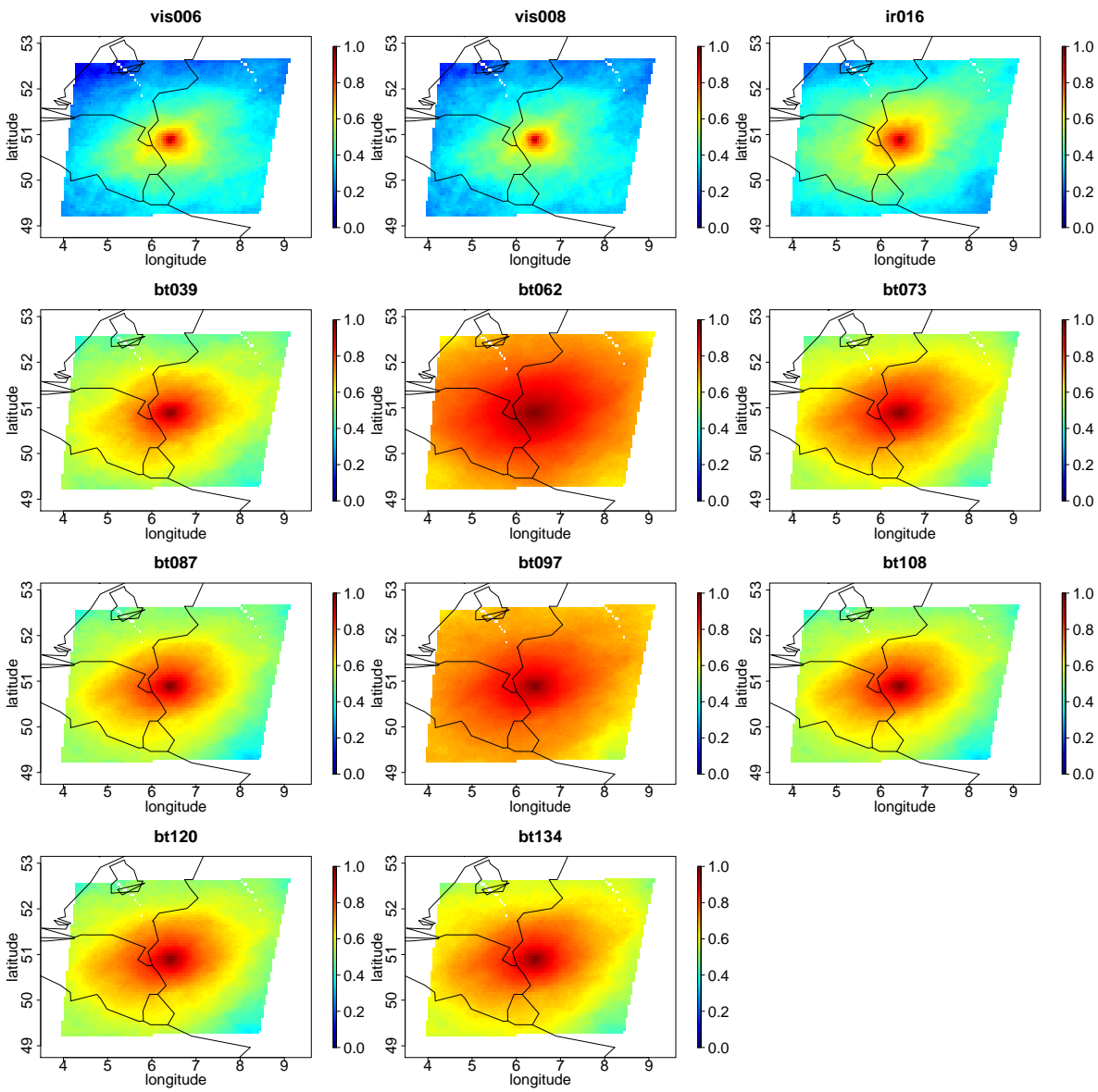

Figure 3. Correlation between time series of SEVIRI pixels and the one nearest to Jülich for all channels. Only cloudy cases from the year 2012 were taken into account.

of the scene or there are many small and separated clouds. These separated clouds might differ largely in cloud height. Thus the correlation is considerable smaller in Fig. 4. An exception is the $3.9 \mu \mathrm{m}$ channel, but the weak decrease of correlation for different cloud covers might arise from saturation of this sensor. This is confirmed by filtering for cold/dark (brightness temperature lower than $280 \mathrm{~K}$ ) and warm/bright (brightness temperature higher than $280 \mathrm{~K}$ ) clouds, respectively. The decrease of correlation with distance is clearly lower for warm clouds (which might be saturated) than for cold clouds.

Too see more details, the correlations around Jülich are shown as a function of distance in Fig. 5 for total cloud cover in red and half covered in blue. The distance was limited to $200 \mathrm{~km}$. For larger distances some patterns in the distribution occur that arise from incomplete circles with radius larger than ca. $200 \mathrm{~km}$ around the reference in the rectangular cutout shown in Figs. 3 and 4.

The range of distances was divided into bins of $20 \mathrm{~km}$ and for each bin the mean correlation and its standard deviation (SD) are calculated. To these mean values a regres- sion curve of type $1-\operatorname{dist}^{b} / a$ is fitted (solid red line for total cloud cover and solid blue line for half covered scenes respectively). 1/SD are used as weighting factors for each distance bin to draw the fit nearer to points with small standard deviations. For larger distances the correlation becomes less significant and shall have less influence on the regression. Additionally the distance where the fitted correlation drops to 0.9 (horizontal dotted line) is given both as the vertical dotted lines and in numbers. It is calculated by dist $0.9=(0.1 * a)^{1 / b}$.

As already said, the correlation decreases with distance. Especially in the visible and near infrared the decrease is quite rapid in the first $10 \mathrm{~km}$, but gets less steep after that. This effect is even stronger for half covered scenes. The decrease in the first kilometre is steeper for half covered than for totally covered scenes but for larger distances the fitted lines are nearly parallel. Correlations above 0.9 are only found in an approximately $1 \mathrm{~km}$ radius around the measurement point for both cases. Instantaneous measurements of cloud optical properties at Jülich are thus only representative for a similar radius though the size of this radius strongly depends on the definition of the chosen threshold of 0.9. Com- 

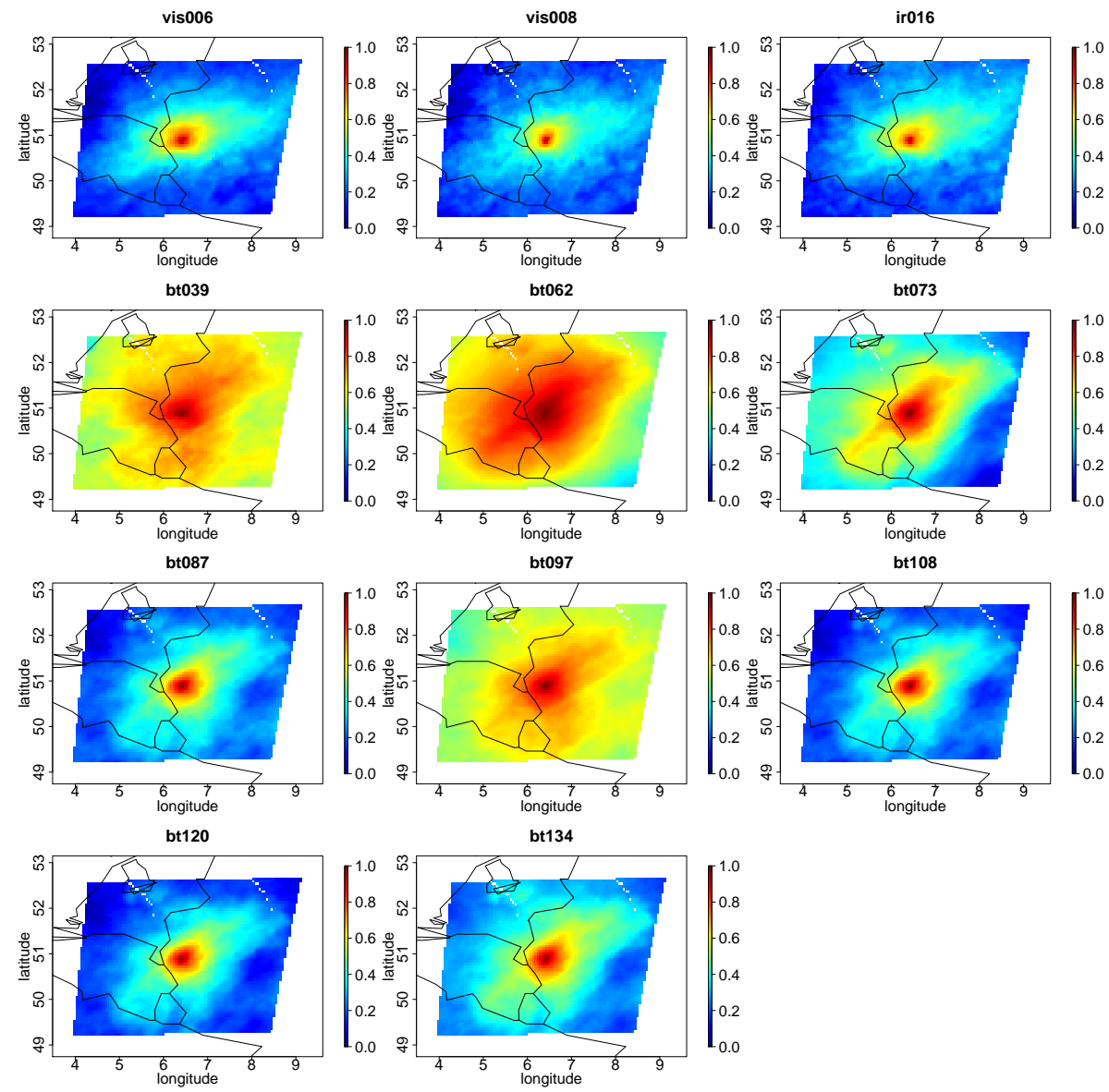

Figure 4. Same as Fig. 3 but for half covered cases from the year 2012.

parisons between ground- and satellite-based measurements in the short-wave range are thus only reasonable for the one pixel nearest to the ground station and depending on the synoptic situation even this might differ, since the calculated radius is less than the pixel size of SEVIRI, making comparisons nearly impossible.

Measurements that involve brightness temperature values like cloud bottom temperature from the ground or cloud top temperature from satellites are somewhat more representative for total cloud cover. In these cases their representativeness reaches to a distance of several $10 \mathrm{~km}$ for total cloud cover. For partly cloudy cases the representativeness of measurements in the long wave is not much higher than in the short-wave range. As said above the likelihood of different cloud layers with different heights increases for partly cloudy scenes in comparison to scenes with total cloud cover. The mentioned exception for channel $3.9 \mu \mathrm{m}$ is also visible in this plot though saturation effects are more likely than a physical meaning.

Channels 6.2, 7.3 and 9.7 $\mu \mathrm{m}$ have high correlations at distances of about $7 \mathrm{~km}$ in case of half covered scenes. This distance increases to nearly $50 \mathrm{~km}$ for $6.2 \mu \mathrm{m}$ and total cloud cover. But these channels give little information on the distribution of clouds since they are additionally influenced by water vapour and ozone, respectively. Both atmospheric components are located above the cloud and thus not or at least less visible from the ground when a cloud is present. Hence, the channels in question cannot be used for an estimation of the representativeness of ground-based measurements of cloud properties. The decrease of correlation for partly covered scenes indicates that clouds still influence these channels but not at the same amount as the other long-wave channels.

A further comparison of the different coefficients for all channels and stations is displayed in Fig. 6. On the left side the spectral distribution of the parameter $a$ and $b$ as well as the correlation length dist 0.9 (from top to bottom) are shown for half covered scenes and on the right side the same is displayed for full cloud cover. The spectral distribution already discussed for Fig. 5 is similar for all stations, though there are some differences to note.

What attracts some attention is the low correlation for Potenza visible in all panels of Fig. 6. All other stations have a similar behaviour especially for dist 0.9 . Potenza is the only 


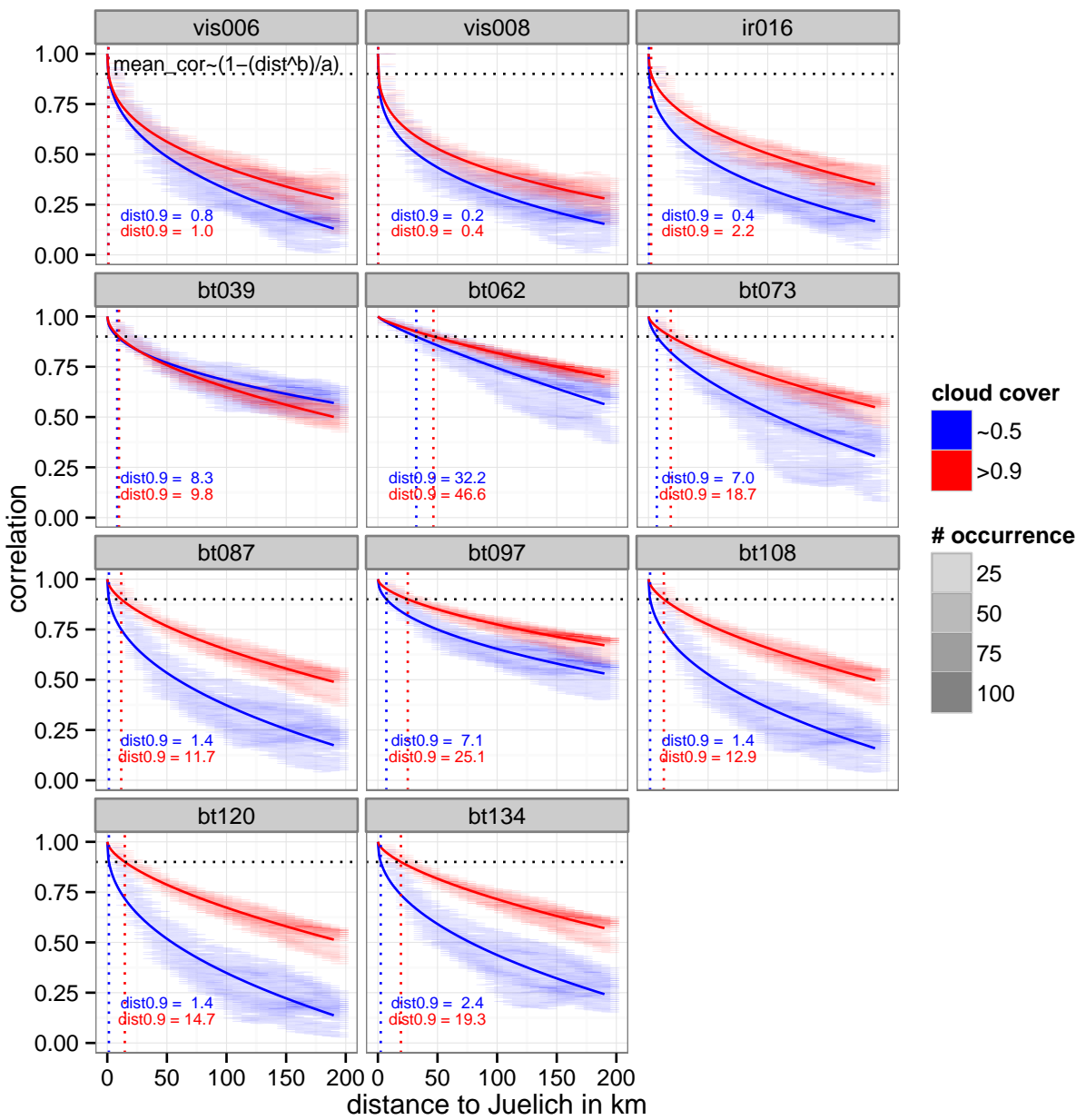

Figure 5. Correlation around Jülich in dependency of the distance for all SEVIRI channels. Red for total and blue for half cloud cover.

station that is not located in the temperate zone with passing cyclones and anticyclones but in the winter rain region of the subtropics. Even the peak in dist 0.9 for $9.7 \mu \mathrm{m}$ (ozone) is missing in case of full cloud cover. A possible reason might be, that on average total ozone concentrations decrease with increasing distance to the polar regions (p. 89 Meul, 2013), resulting in a weaker ozone absorption and stronger influence of tropospheric clouds on the observed brightness temperatures.

Further, dist 0.9 depends on the combination of both coefficients, though $a$ seems to have the larger influence since its spectral distribution resembles that of dist 0.9 some more especially for total cloud cover. As said before, $b$ describes the curvature of the correlation as a function of distance with $b=1$ in a linear case, a convex shape for $b>1$ and concave for $b<1$, respectively. In most cases, except for some stations in the case of half cloud cover, the decrease of correlation with distance is sub-linear. The most linear cases are the water vapour channels. The ozone channel has a peak in coefficient $a$, but a local minimum in $b$ with an exception for the station Mace Head. Altogether the water vapour and ozone channels show the largest correlation length although this maximum is higher when the cloud layer below is dense. These features can also be explained by regarding the SEVIRI weighting functions (Schmetz et al., 2002).

Those channels that respond to cloud properties exhibit smaller correlation lengths which even shrink when the cloud cover decreases. A detailed analysis for which station the correlation length is high or low and which cloud types or properties cause these differences is complicated. The differences between the single stations according to frequent synoptic situations as described in Sect. 2 are hard to see in the results especially since the order of the stations in dependence of their correlation length varies with the cloud cover. Palaiseau and Cabauw e.g. have a comparatively large correlation length for the window channels in case of total cloud cover, but for half covered cases the correlation length for Leipzig is larger. Here a more detailed deviation of different cloud types in the input data would be needed, but therefore we would need a larger database to get a sufficient number of all different cases. 


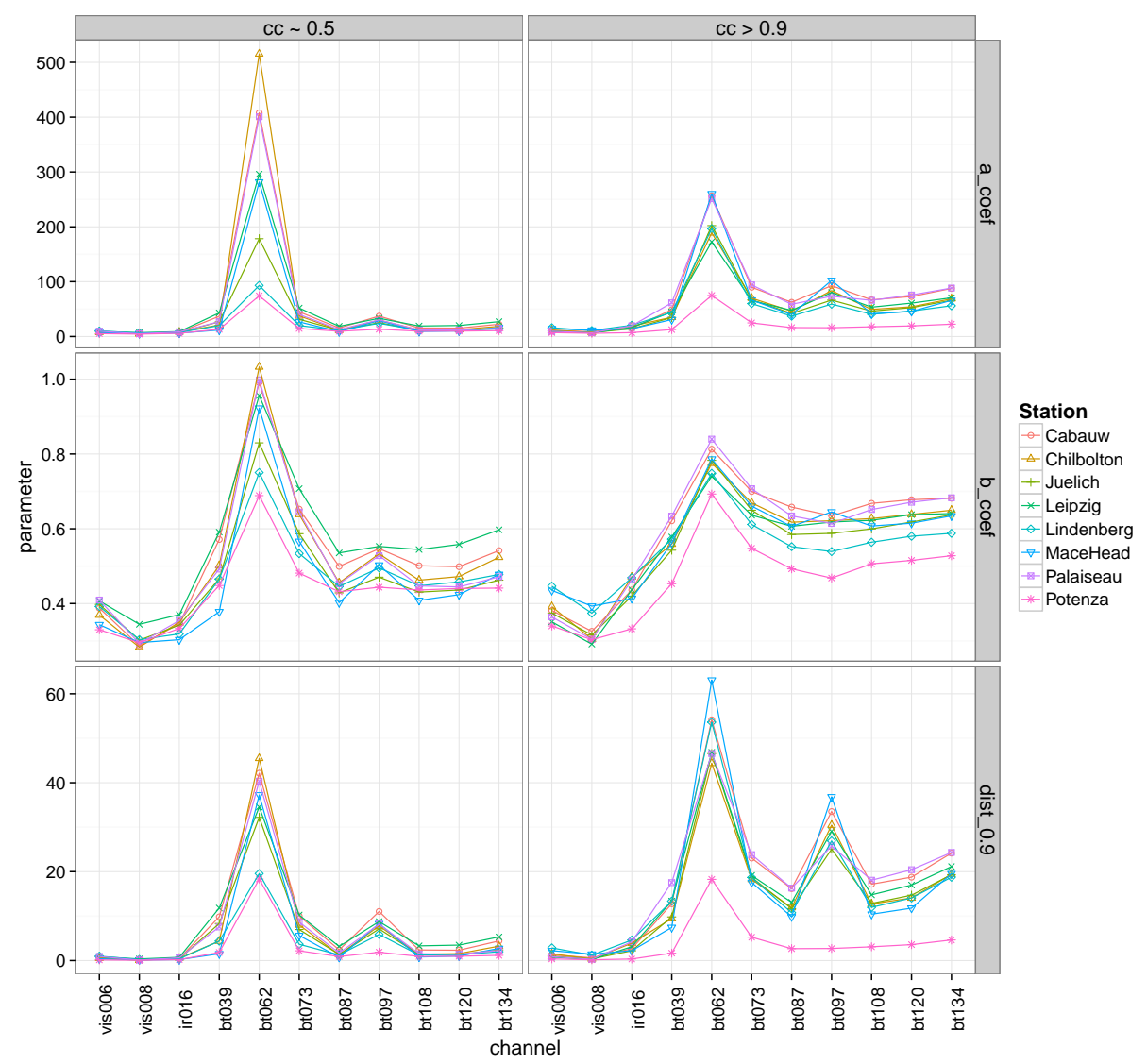

Figure 6. Comparison of coefficients $a$ (upper panel) and $b$ (middle panel) as well as dist 0.9 (lower panel) for two different cloud covers (left side: half covered scenes; right side: fully covered scenes) and for all channels ( $x$ axes) and locations (different colours, see legend).

To estimate the comparability of forecast models and ground-based stations the correlation at distances typical for the size of model grid boxes were summarized in Fig. 7. As before on the left side the correlations at 2.8, 7,28, and $70 \mathrm{~km}$ (top to bottom) are shown for half cloud cover and on the right side for total cloud cover. With decreasing cloud cover and increasing model grid size or distance to the ground station respectively the correlation decreases and the features of the different channels become more distinct. Comparisons between predicted cloud properties in a single grid box of a regional weather model like COSMO-DE or COSMO-EU to the corresponding measured values at a ground station should be possible especially for cloud top temperatures. For cloud optical properties the differences might be larger, but a comparison is still useful. For global climate models like GFS the comparison of cloud properties becomes more difficult especially for cloud optical properties in case of not fully covered scenes because the correlation is in this case $\sim 0.6$. For totally covered scenes a comparison of cloud temperatures should still be reasonable despite the large grid size of GFS since the correlation is still around 0.8 .

\section{Influence of timescales}

Clouds are highly variable not only in space like the different results for complete and partial cloud cover have shown, but also in time. The former results are all based on data from each 15 min time step of SEVIRI, while the output of forecast models is normally only given every few hours. To get information about the correlation length for larger time steps the methodology from the previous sections was repeated for daily means of the SEVIRI data. This means for the totally covered cases, that the daily mean of the cloud cover for each pixel in the investigation area must be larger than 0.9 to be selected for the analysis. Thus we have a totally overcast day and the results will show how strongly the inner structures within this large cloud system are smoothed out during the passage over the station. For partly covered scenes there are different possibilities. One possibility is that for the first half of the day the whole scene was covered and then the clouds dissolve or the other way around, half of the day was clear until clouds developed. The second possibility is that half of the area was overcast the whole day and the other half was clear (like at a stationary boundary of air masses). It is also possible that the whole area was covered by variable clouds 


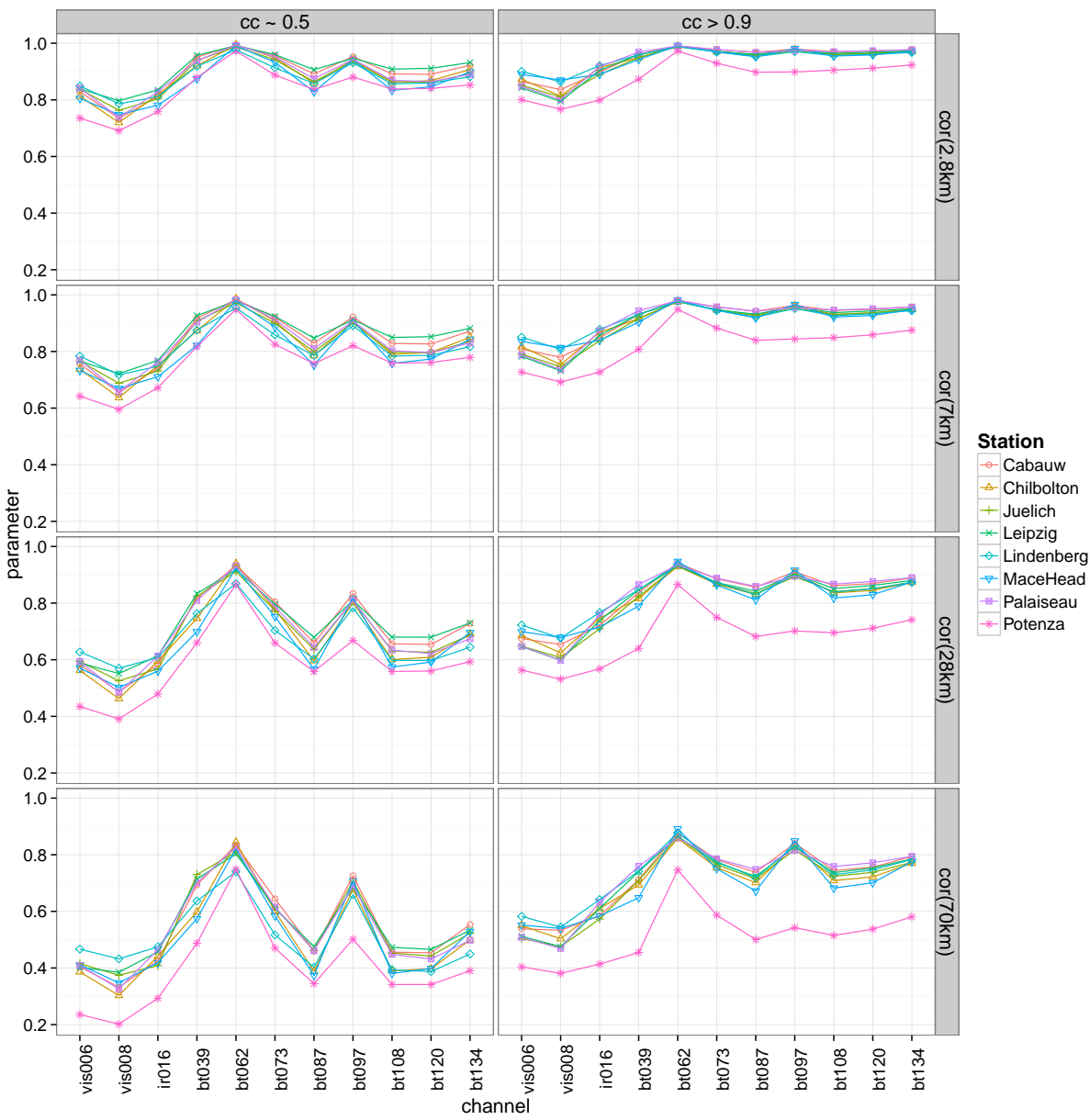

Figure 7. Comparison of correlation at distances that correspond to typical grid-sizes of forecast models (from top panel to bottom) for two cloud covers (left side: half covered scenes; right side: fully covered scenes) and for all channels ( $x$ axis) and location (different colours, see legend).

for the whole day e.g. after the passage of a cold front. At the moment the different possibilities are not separated from each other and all contribute to the following results. In comparison to the previous sections the database encompasses now between 25 (Potenza) and 80 (German stations) cases of total cloud cover and 40 to 90 cases of half covered days from a total of 366 days.

Since the purpose of this part of the study is to estimate the comparability of forecasts with mean values of groundbased data only corresponding images to Fig. 7 are shown in Fig. 8. As expected the features of single clouds or of structures within one large cloud system moving across the examination area in one day are smoothed out for the mean values causing an increase of the correlations at the different distances. The differences for the single stations almost disappear for regional weather models and total cloud cover except for Potenza, which is still less correlated with its surroundings in the case of short-wave measurements. A comparison to averaged ground-based data is possible with good accuracy for both amounts of cloudiness. For global models with a resolution of approximately $28 \mathrm{~km}$ the comparisons to ground-stations should also be reasonable for time intervals of several hours. But for larger grid-boxes the differences to ground-based measurements will be noticeable especially for variable cloud cover.

Additionally time series from 12:00 UTC only are examined. That corresponds to models that generate output for a specific time stamp every few hours. Another reason for this experiment was to eliminate a possible influence from a daily cycle. The results show no larger difference to the results of all single time steps from Sect. 3. It can be concluded, that there is no influence by a daily cycle in the upper results and that the model output for instantaneous time steps can be compared as well with ground based data as satellite data.

\section{Conclusions and outlook}

The correlations between time series of SEVIRI observations for $300 \mathrm{~km} \times 300 \mathrm{~km}$ area surrounding eight ground- 


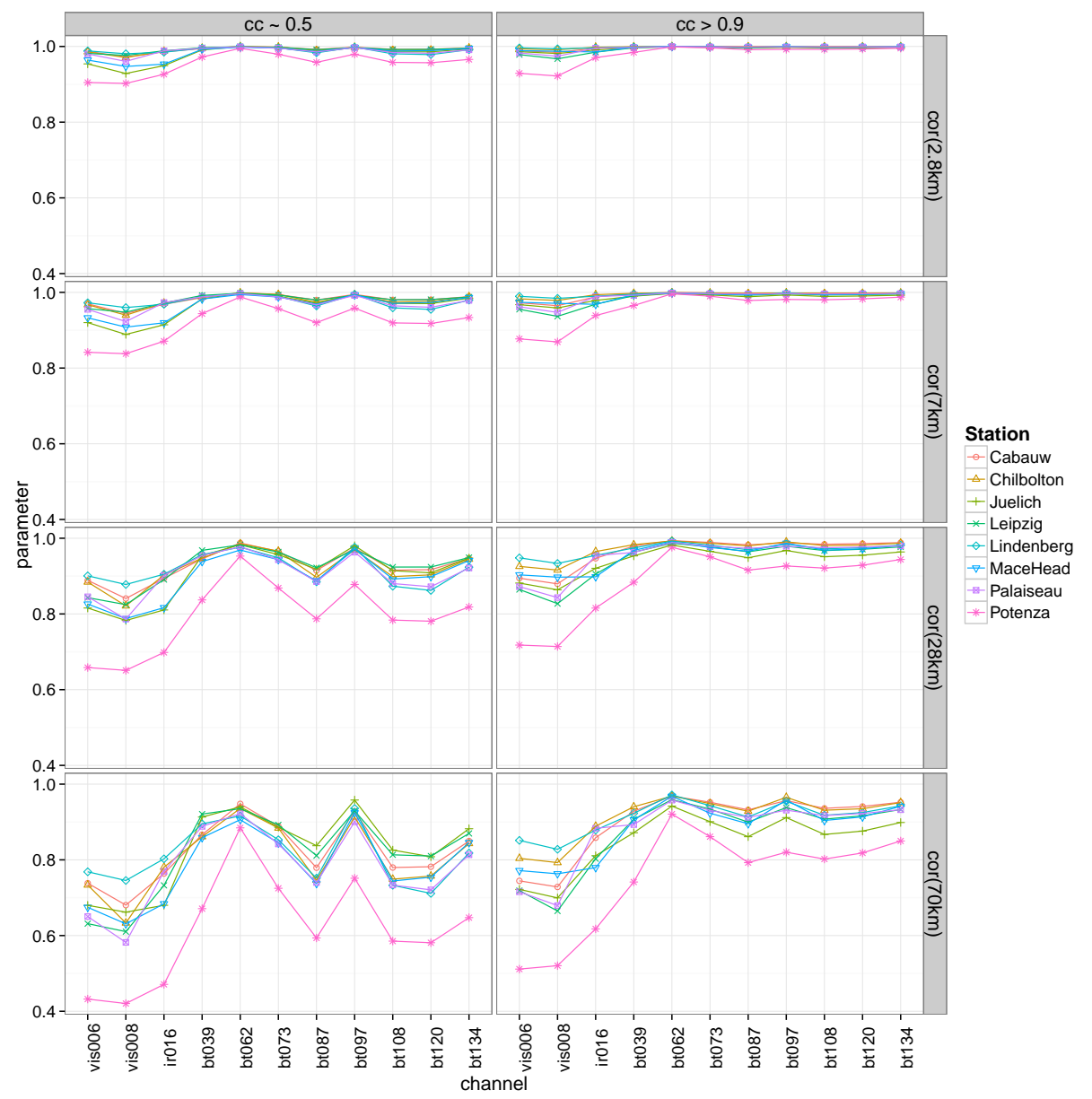

Figure 8. Same as Fig. 7 but for daily means of SEVIRI data.

based measurement sites have been examined during this study. The comparisons have been carried out for two different amounts of cloud cover. With the help of the correlations a representativeness of these measurement sites with respect to their surrounding area was estimated for full and half cloud cover, respectively. To eliminate the influence of varying surface albedo, clear sky pixels are left out for both cases. By defining the 0.9 correlation radius dist 0.9 the representativeness of measurements in different wavelength regimes and locations are compared. The following values are strongly depending on this definition and if less accuracy is required, a radius for a lower threshold than 0.9 might be defined and the following values for the estimated representativeness would increase.

Measurements in the visible and near infrared range were found to be representative for an area with a radius of about 1 to $4 \mathrm{~km}$ for instantaneous measurements in case of total cloud cover. For half covered scenes these values decrease slightly, but since the correlation radius is already smaller than the pixel size of MSG it just indicates the difficulties of an accurate comparison between satellite data and ground-based measurements, especially for cloud mi- crophysical properties. For measurements in the infrared the correlation length increases to about $20 \mathrm{~km}$. An exception is the correlation around Potenza in southern Italy. The representativeness seems to be much lower there (only for an area of approximately $10 \mathrm{~km}$ in the infrared). The differences between partly and full cloud cover becomes more pronounced in these cases since the correlation lengths are high and thus the differences can also become larger. Besides, the probability that complete cloud cover consists of one cloud layer with a similar top height is larger than the likelihood of similar top heights in a partly covered scene that consists of several clouds and cloud layers. The highest correlations were found for channels that are influenced by structures above the clouds, i.e. water vapour and ozone. The correlation lengths of these channels reaches values of up to $40-60 \mathrm{~km}$, but those results are not transferable to the ground-based measurements since they do not see water vapour and ozone above the cloud. Despite this, the effect of the underlying clouds is still visible when different cloud covers are compared.

To estimate the comparability of ground measurements to model grid-boxes, the correlations at distances typical for model grid-sizes were compared. The results show a good 
comparability for regional weather models with grid sizes smaller than $\sim 10 \mathrm{~km}$. For global models with larger grid sizes the comparisons would become less accurate since correlations at $28 \mathrm{~km}$ distance to a ground station decrease below 0.8 for long-wave measurements and to 0.6 in the short-wave range.

The representativeness or comparability can be strongly increased by utilizing daily means for each pixel instead of instantaneous measurements. Since the output of weather models already has a lower temporal resolution than measurements, this fact is helpful for actual comparisons between models and real data. The correlations increase to nearly 1.0 for regional models and seldom fall below 0.8 . The reason for the increase of correlation is the smoothing of horizontal cloud structures that pass over the whole scene during one day.

A verification of the results of this study with groundbased measurements would be desirable but quite complicated to achieve since a larger number of stations would be needed to get area-wide ground-based data. Still there are some ideas for further investigations based on satellite data. This study shows the representativeness of ground-based measurements of cloud properties within a radius of $200 \mathrm{~km}$ around the stations. This area could, in a further study, be divided into the four cardinal directions to estimate the influence of the main wind direction. Figure 3 already reveals slightly lower correlation in the north western corner of the cutout around Jülich in comparison to the other three corners. Furthermore the data might also be further separated by the synoptic conditions. Especially for an overpass of a frontal zone we should see an influence caused by the wind direction and the partly covered cases could be divided into cases with variable cloud cover and cases with a sharp boundary between a large scale cloud and clear sky across the examination area. For convective days a time step in the morning could be compared to a time step in the afternoon to investigate whether cloud formation could be seen in the correlation length. Therefore an examination of the correlations under clear sky conditions would be helpful. Another reason for the importance of clear sky examinations is that for thin clouds the satellite also receives reflected radiance from the surface and its homogeneity or inhomogeneity might cause some uncertainties into the results for total cloud cover. Additionally the clear sky correlations can be compared to land use and elevation data. A first examination of clear sky cases resulted in a clear division of the examined stations in those with surroundings that include large fraction of water bodies and those merely surrounded by land.

For a further separation of cloudy cases according to cloud top height, (optical) thickness and homogeneity or synoptic situation a larger amount of data would be desirable. Instead of data from only one year the entire operation time of SEVIRI instruments on the different MSG satellites could be used. That would be the time period from 2004 to 2013 so far.
For clouds of smaller scale the high-resolution visible (HRVIS) channel might be utilized. This channel would also help to verify the results of the method described in this paper especially for those cases where the calculated correlation radius is smaller than the SEVIRI pixel size.

Acknowledgements. This study was done in context of the project Integrated Cloud Observations from Ground and Space - A Way to Combine Time and Space Information (ICOS) funded by the German Research Foundation (Deutsche Forschungsgesellschaft DFG) under grant CR 111/8-1. Thanks to all our colleagues, especially André Hollstein and Cintia Carbajal Henken, who gave their advice for the successful development of this study. The authors are very thankful for the helpful and appreciated comments from the reviewers and people in the discussion forums.

Edited by: B. Mayer

\section{References}

Boehme, T., Stapelberg, S., Akkermans, T., Crewell, S., Fischer, J., Reinhardt, T., Seifert, A., Selbach, C., and Van Lipzig, N.: Longterm evaluation of COSMO forecasting using combined observational data of the GOP period, Meteorol. Z., 20, 119-132, 2011.

Bouniol, D., Protat, A., Delanoë, J., Pelon, J., Poriou, J.-M., Bouyssel, F., Tompkins, A. M., Wilson, D. R., Morielle, Y., Haeffelin, M., O'Connor, E. J., Hogan, R. J., Illingworth, A. J., Donovan, D. P., and Baltink, H.-K.: Using Continuous Ground-Based Radar and Lidar Measurements for Evaluating the Representation of Clouds in Four Operational Models, J. Appl. Meteor. Climatol., 49, 1971-1991, 2010.

Cahalan, R. F., Short, D. A., and North, G. R.: Cloud Fluctuation Statistics, Mon. Weather Rev., 110, 26-43, 1982.

Crewell, S., Mech, M., Reinhardt, T., Selbach, C., Betz, H.-D., Brocard, E., Dick, G., O'Connor, E., Fischer, J., Hanisch, T., Hauf, T., Hünerbein, A., Delobbe, L., Mathes, A., Peters, G., Wernli, H., Wiegner, M., and Wulfmeyer, V.: The general observation period 2007 within the priority program on quantitative precipitation forecasting: Concept and first results, Meteorol. Z, 17, 849866, 2008.

Deneke, H. M., Knap, W. H., and Simmer, C.: Multiresolution analysis of the temporal variance and correlation of transmittance and reflection of an atmospheric column, J. Geophys. Res., 114, D17206, doi:10.1029/2008JD011680, 2009.

Ebell, K., Orlandi, E., Hünerbein, A., Löhnert, U., and Crewell, S.: Combining ground-based with satellite-based measurements in the atmospheric state retrieval: Assessment of the information content, J. Geophys. Res. Atmos., 118, 6940-6956, 2013.

Feldmann, H., Früh, B., Schädler, G., Panitz, H.-J., Keuler, K., Jacob, D., and Lorenz, P.: Evaluation of the precipitation for Southwestern Germany from high resolution simulations with regional climate models, Meteorol. Z., 17, 455-465, 2008.

Greuell, W. and Roebeling, R. A.: Toward a Standard Procedure for Validation of Satellite-Derived Cloud Liquid Water Path: A Study with SEVIRI Data, J. Appl. Meteor. Climatol., 48, 15751590, 2009. 
Grützun, V., Quaas, J., Morcette, C. J., and Ament, F.: Evaluating statistical cloud schemes: What can we gain from ground-based remote sensing?, J. Geophys. Res. Atmos., 118, 10507-10517, 2013.

Hamann, U., Walter, A., Bennartz, R., Thoss, A .and Meirink, J. F., and Roebeling, R.: Inter-comparison of cloud detection and cloud top height retrievals using the CREW database, AIP Conference Proceedings, 1531, 460, 2013.

Illingworth, A. J., Hogan, R. J., O’Connor, E. J., Bouniol, O., Delanoë, J., Pelon, J., Protat, A., Brooks, M. E., Gaussiat, N., Wilson, O. R., Donovan, D. P., Klein Baltink, H., van Zadelhoff, G.-J., Eastment, J. D., Goddard, J. W. F., Wrench, C. L., Haeffelin, M., Krasnov, O. A., Russchenberg, H. W. J., Piriou, JM.and Vinit, F., Seifert, A., Tompkins, A. M., and Willén, U.: Cloudnet Continuous Evaluation of Cloud Profiles in Seven Operational Models Using Ground-Based Observations, B. Am. Meteorol. Soc., 88, 883-898, 2007.

IPCC: Summary for Policymakers, in: Climate Change 2013: The Physical Science Basis. Contribution of Working Group I to the Fifth Assessment Report of the Intergovernmental Panel on Climate Change, Cambridge Univ. Press, 2013.

Kiehl, J. T. and Trenberth, K. E.: Earth's Annual Global Mean Energy Budget, B. Am. Meteorol. Soc., 78, 197-208, 1997.

Long, C. N. and Ackerman, T. P.: Surface measurements of solar irradiance: A study of the spatial correlation between simultaneous measurements at separated sites, J. Appl. Meteor., 34, 10391046, 1995.

METEK: Cloud Radar MIRA-35, available at: http://metek.de/wp-content/uploads/2014/05/

Metek-Scanning-Doppler-Cloud-RADAR-MIRA-35-Datasheet. pdf (last access: 29 January 2015), 2014.

Meul, S.: Ozon-Klima-Wechselwirkungen in Simulationen mit dem Klima-Chemie-Modell EMAC, PhD thesis, Freie Universität Berlin, 2013.
Pfeifer, M., Yen, W., Hagen, M., Craig, G., Reinhardt, T., Mech, M., Crewell, S., Huenerbein, A., Schroeder, M., Fischer, J., Baldauf, M., and Seifert, A.: Validating precipitation forecasts using sensor synergy: A case study approach, Meteorol. Z., 19, 601-617, 2010.

Reuter, M., Thomas, W., Albert, P., Lockhoff, M., Weber, R., Karlsson, K.-G., and Fischer, J.: The CM-SAF and FUB Cloud Detection Schemes for SEVIRI: Validation with Synoptic Data and Initial Comparison with MODIS and CALIPSO, J. Appl. Meteor. Climatol., 48, 301-316, 2009.

Schmetz, J., Pili, P., Tjemkes, S., Just, D., Kerkmann, J., Rota, S., and Ratier, A.: An Introduction to Meteosat Second Generation (MSG), B. Am. Meteorol. Soc., 83, 977-992, 2002.

Schutgens, N. A. J. and Roebling, R. A.: Validating the Validation: The Influence of Liquid Water Distribution in Clouds on the Intercomparison of Satellite and Surface Observations, J. Atmos. Oceanic Technol., 26, 1457-1474, 2009.

Welch, R. M. and Wielicki, B. A.: Stratocumulus Cloud Field Reflected Fluxes: The Effect of Cloud Shape, J. Atmos. Sci., 41, 3085-3103, 1984.

Whitlock, C. H., Hay, J. E., Robinson, D. A., Cox, S. K., Wardle, D. I., and LeCroy, S. R.: Downward surface irradiance from 17 sites for the FIRE/SRB Wisconsin Experiment from Oct. 12 through Nov. 2, NASA Technical Manual 102596, p. 272, 1990.

Wiegner, M. and Geiß, A.: Aerosol profiling with the Jenoptik ceilometer CHM15kx, Atmos. Meas. Tech., 5, 1953-1964, doi:10.5194/amt-5-1953-2012, 2012.

Zinner, T. and Mayer, B.: Remote sensing of stratocumulus clouds: Uncertainties and biases due to inhomogeneity, J. Geophys. Res., 111, D14209, doi:10.1029/2005JD006955, 2006. 